\title{
Prevalência e perfil de sensibilidade aos antimicrobianos de microrganismos isolados de secreções de pele em um hospital escola
}

\section{Prevalence and profile of antimicrobial sensitivity of microrganisms isolated from skin secretions in a school hospital}

Taciéli Fagundes da Rosa, Mônica de Abreu Rodrigues, Fernanda Aguirre Carvalho, Vitória Segabinazzi Foletto, Marissa Bolson Serafin, Angelita Bottega, Laísa Nunes Franco, Bruno Rafael de Paula, Divanir Maria Acosta Fagundes, Rosmari Hörner

\author{
Como citar este artigo: \\ DA ROSA, TACIÉLI F; RODRICUES, \\ MÔNICA A.; CARVALHO, \\ FERNANDA A.; FOLETTO, VITÓRIA \\ S.; SERAFIN, MARISSA B.; BOTTECA, \\ ANCELITA; FRANCO, LAÍSA N.; \\ DE PAULA, BRUNO RAFAEL; \\ FACUNDES, DIVANIR M. A.; HÖRNER, \\ ROSMARI; Prevalência e perfil de \\ sensibilidade aos antimicrobianos \\ de microrganismos isolados de \\ secreções de pele em um hospital \\ escola. Revista Saúde (Sta. \\ Maria). 2020; 46 ( I).
}

\section{Autor correspondente: \\ Nome: Taciéli Fagundes da Rosa E-mail: tacielifagundesdarosa@ gmail.com \\ Telefone: (55) 999252657 \\ Formação Profissional: Formada em Farmácia e Mestra em Ciências Farmacêuticas pela Universidade Federal de Santa Maria (UFSM) que fica na cidade de Santa Maria, Rio Grande do Sul, Brasil.}

Filiação Institucional: Universidade Federal de Santa Maria Endereço para correspondência: Rua: Avenida Roraima, n० 1000 Bairro: Camobi

Cidade: Santa Maria

Estado: Rio Grande do Sul

CEP: 97 105-900

Data de Submissão:

21/01/2020

Data de aceite:

$01 / 04 / 2020$

Conflito de Interesse: Não há conflito de interesse

\section{RESUMO}

OBJETIVO: O objetivo deste trabalho foi avaliar a prevalência e perfil de resistência dos microrganismos isolados de secreções de pele e tecidos moles. Este tipo de estudo, pelo que é de nosso conhecimento, foi o primeiro realizado neste hospital com período de quatro anos e elevado número de amostras. MÉTODOS: Realizou-se um estudo observacional retrospectivo de culturas de infecções de pele coletadas em hospital escola terciário, no período de janeiro de 2014 a dezembro de 2017. Os testes de identificação dos microrganismos e perfil de sensibilidade foram efetuados por meio de sistemas convencionais e automatizados (Vitek® 2 - bioMérieux). RESULTADOS: No período deste estudo, 675 amostras resultaram positivas, sendo Staphylococcus aureus (118; 17,48\%), Pseudomonas aeruginosa $(106 ; 15,70 \%)$, Staphylococcus epidermidis $(65 ; 9,63 \%)$ e Escherichia coli $(60 ; 8,89 \%)$ os microrganismos predominantes. Em relação à Pseudomonas aeruginosa, 53,77\% das cepas apresentaram resistência ao imipenem e $44,34 \%$ ao meropenem. As opções terapêuticas para o tratamento das infecções causadas por esse microrganismo são limitadas. CONCLUSÕES: O perfil epidemiológico dos agentes envolvidos é similar ao citado na literatura internacional, o que diferencia é o perfil de sensibilidade. Fato que necessita monitoramento é que Pseudomonas aeruginosa ocupou o segundo lugar na etiologia e apresentou significativa resistência frente aos carbapenêmicos.

PALAVRAS-CHAVE: Infecção Hospitalar; Ferida Cirúrgica; Infecção dos Ferimentos; Procedimentos Cirúrgicos Ambulatórios; Pele.

\section{ABSTRACT}

OBJECTIVE: The objective of this work was to evaluate the prevalence and resistance profile of microorganisms isolated from skin and soft tissue secretions. This type of study, as far as we know, was the first carried out in this hospital with a period of four years and a large number of samples. METHODS: A retrospective observational study of cultures of skin infections collected in a tertiary teaching hospital was carried out from January 2014 to December 2017. The tests for identification of the microorganisms and sensitivity profile were carried out using systems conventional and automated systems (Vitek® 2 - bioMérieux). RESULTS: During the period of this study, 675 samples were positive, being Staphylococcus aureus (118; 17.48\%), Pseudomonas aeruginosa (106; 15.70\%), Staphylococcus epidermidis $(65 ; 9,63 \%)$ and Escherichia coli $(60 \%)$ were positive in the study period $(60 ; 8.89 \%)$ the predominant microorganisms. Regarding Pseudomonas aeruginosa, $53.77 \%$ of the strains had resistance to imipenem and $44.34 \%$ to meropenem. Therapeutic options for the treatment of infections caused by this microorganism are limited. CONCLUSION: The epidemiological profile of the agents involved is similar to that reported in the international literature, which differentiates the sensitivity profile. A fact that needs monitoring is that Pseudomonas aeruginosa occupied the second place in the etiology and presented significant resistance against the carbapenems.

KEYWORDS: Hospital Infection; Surgical wound; Wound Infection; Ambulatory Surgical Procedures; Skin. 


\section{INTRODUÇÃO}

Os microrganismos que habitam a pele e mucosas estão entre os principais agentes etiológicos que causam as Infecções Hospitalares (IH). Estas, muitas vezes são favorecidas pela utilização de dispositivos médicos e por práticas de higienização inadequadas ou insuficientes por parte dos profissionais de saúde ${ }^{1}$. Tais infecções constituem questões de saúde pública e por muitas vezes apresentam um caráter prevenível, podendo ser evitadas com ações simples, como a lavagem das mãos, até ações mais complexas, como a realização de uma cirurgia com material adequadamente esterilizado, podem impedir sua ocorrência².

Grande parte dos pacientes hospitalizados são afetados por infecções de pele e tecidos moles, as quais são comuns nos cuidados da emergência, sendo por muitas vezes difícil a diferenciação entre os casos que requerem intervenção imediata daqueles menos severos. Entre as infecções de pele, as mais comumente relatadas na literatura são as provenientes de secreção de ferida operatória (sítio cirúrgico), líquido de abscesso, escaras e secreções de membros inferiores ${ }^{3,5}$.

Infecção de Sítio Cirúrgico (ISC) é uma das principais Infecções Relacionadas à Assistência à Saúde (IRAS) no Brasil, ocupando a terceira posição entre todas as infecções ocorridas em serviços de saúde e a segunda posição entre os pacientes hospitalizados ${ }^{4}$. Estima-se que estas podem ser evitadas em até $60 \%$ dos casos, através da aplicação das medidas de orientação e prevenção recomendadas em manuais e diretrizes. As ISCs ocorrem em aproximadamente meio milhão de pacientes na América do Norte anualmente ${ }^{5}$. Dados recentes revelam que nos Estados Unidos comprometem de $2 \%$ a $5 \%$ dos pacientes submetidos aos procedimentos cirúrgicos, e que entre 160.000 a 300.000 episódios de ISCs ocorrem a cada ano no país ${ }^{6}$.

Assim, longe de estar acabada a luta contra as infecções hospitalares, em destaque para infecções de pele, torna-se, agora, ainda mais severa e difícil, pois o uso indiscriminado de antibióticos tem aumentado consideravelmente o número de diferentes espécies bacterianas resistentes aos antimicrobianos utilizados normalmente como terapia medicamentosa'. O meio hospitalar constitui um vasto e excelente habitat para bactérias adquirirem resistência aos antibióticos. De um modo geral, o paciente internado está imunodeprimido e sujeito a diversas terapias medicamentosas e/ou invasivas, que o torna susceptível a adquirir infecção hospitalar.

\section{OBJETIVOS}

Sendo o nosocômio em estudo um hospital escola terciário que recebe pacientes de vários municípios e processa um significativo número de amostras provenientes de secreções de pele, torna-se essencial o conhecimento de dados obtidos pelas análises dessas secreções. Sendo assim, o objetivo deste trabalho foi avaliar a prevalência bem como 0 perfil de sensibilidade aos antimicrobianos dos microrganismos envolvidos nessas infecções. 


\section{MÉTODO}

O estudo foi aprovado pelo Comitê de Ética em Pesquisa da Universidade Federal de Santa Maria sob o $\mathrm{n}^{0}$ 38850614.4.0000.5346.

Foi realizado um estudo observacional retrospectivo de culturas de secreções de pele solicitadas em hospital escola terciário, no período de janeiro de 2014 a dezembro de 2017. Os dados se referiram a registros laboratoriais de exames bacteriológicos coletados de pacientes internados e atendidos no nosocômio, no período estudado em que houve solicitação médica de cultura microbiológica de secreções de pele. As informações coletadas se referiram a gênero (masculino/feminino), setor ou clínica do hospital onde foi registrado ou internado cada paciente, resultado da cultura, microrganismo identificado e perfil de resistência frente aos antimicrobianos.

Os exames foram realizados conforme o Procedimento Operacional Padrão (POP) do laboratório, formulado com base nos critérios da Agência Nacional de Vigilância Sanitária, Clinical Laboratory Standards Institute (CLSI) e Infectious Diseases Society of America (IDSA) conforme descrito a seguir: a coleta das amostras clínicas aconteceu preferencialmente por aspiração em frasco estéril ou seringa e foi realizada por médicos e/ou enfermeiros da instituição $0^{4,12,6}$. Quando não foi possível ou viável para o quadro do paciente ser realizado esse procedimento, foram aceitas coletas realizadas com swabs. Nestas, foi feita uma antissepsia rigorosa eliminando crostas e secreções superficiais utilizando soro fisiológico estéril. Foi utilizado um swab para fricção local que, em seguida, foi transportado em meio Stuart. Excetuando-se os casos de sítios inguinais e axilar, foram friccionados mais três swabs sem meio de transporte. Também foram aceitos fragmentos de tecido cuja coleta foi realizada por procedimento cirúrgico e encaminhados em frasco estéril e sem conservantes. Todas as amostras foram encaminhadas de imediato para os procedimentos laboratoriais.

Para cada amostra, foram realizados dois esfregaços em lâmina de microscopia utilizando os swabs sem meios de cultura. Foi procedida a coloração dos esfregaços pelo método de Gram. Também foi realizado o exame ao microscópio óptico utilizando apenas a amostra em salina estéril.

Os swabs nasais e inguinais foram encaminhados para pesquisa de portadores de bactérias multirresistentes e foram inoculados em meio sal manitol com disco de cefoxitina e meio tioglicolato. As secreções colhidas por punção e os demais swabs foram inoculados em ágar sangue, MacConkey, Ágar azida e tioglicolato. Posteriormente, foram inoculadas em MacConkey novamente, ágar Brolacin e ágar Thayer Martin (ATM).

Os fragmentos de tecido foram triturados em placa de petri estéril com o auxílio de uma lâmina de bisturi. Foram confeccionados dois esfregaços por imprint, ou seja, pressionando-se os fragmentos triturados sobre a lâmina de microscopia com o auxílio de uma pinça flambada e esfriada. Como não houve solicitação médica explícita para exame de BAAR, as partes cortadas foram apenas semeadas em ágar chocolate, tioglicolato, MacConckey e ágar Brolacin com discos de aztreonam. 
Após as semeaduras em ágar sangue, foram realizadas picadas no meio para favorecer o possível aparecimento de hemólise. Todos os meios foram incubados em estufa de crescimento a $35^{\circ} \mathrm{C} \pm 2{ }^{\circ} \mathrm{C}$ por 18 a 24 horas. 0 ágar sangue foi incubado em microaerofilia e os demais em aerobiose. A partir disso, foi relatado o crescimento microbiano levando em consideração a flora normal e a existência de possíveis contaminantes. Havendo infecção, foram isolados e identificados os patógenos e procedido o antibiograma por disco-difusão.

Para realização do antibiograma, as cepas que até então apresentaram crescimento foram semeadas em meio Mueller-Hinton e foram adicionados discos de antibióticos. A placa foi mantida em estufa de crescimento a $35^{\circ} \mathrm{C} \pm 2$ ${ }^{\circ} \mathrm{C}$ por 18 a 24 horas. E foi estabelecida a sensibilidade/ resistência aos antibióticos pela medida em milímetros do halo formado. Também foram realizadas as identificações e perfis de sensibilidade através de metodologias automatizadas (Vitek® 2 - bioMérieux).

\section{RESULTADO}

No período do estudo foram identificadas 675 culturas de pele e tecidos moles, sendo 415 (61,48\%) de feridas cirúrgicas, 119 (17,63\%) de secreções de escara, 109 (16,15\%) de secreções de abscesso e 32 (4,74\%) de secreções de membros inferiores. Entre as culturas, $379(56,15 \%)$ foram provenientes de amostras de pacientes do gênero masculino. Quanto à unidade clínica de origem de cada paciente, verificamos que a Clínica Cirúrgica foi o setor hospitalar onde houve o maior número de isolamentos (20,89\%), seguida dos Ambulatórios (16,89\%), conforme demonstrado no Gráfico 1.

Gráfico 1. Unidades hospitalares de isolamento das 675 culturas de secreções de pele e tecidos moles isoladas no hospital terciário de janeiro de 2014 a dezembro de 2017.

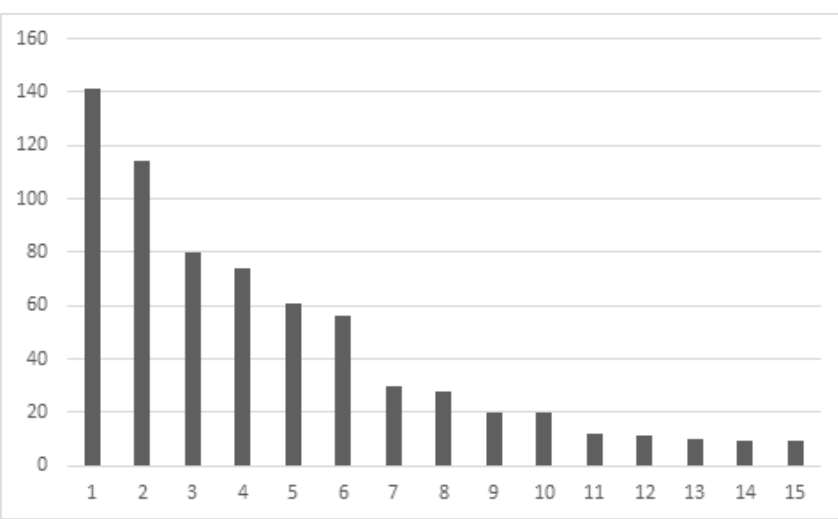

Fonte: Autora, 2020.

1 - Clínica Cirúrgica (20,89\%), 2 - Ambulatório (16,89\%), 3 - Pronto-Atendimento (PA) Adulto (11,85\%), 4 - Bloco Cirúrgico (11,85\%), 5 - Sala de Recuperação (9,04\%), 6 - Setor de Nefrologia (8,30\%), 7 - Unidade de Tratamento Intensivo (UTI) Adulta (4,44\%), 8 - Setor de 
Oncologia (4,15\%), 9 - UTI Pediátrica (2,96\%), 10 - Centro Obstétrico (2,96\%), 11- Sistema de Internação Hospitalar (SID) (1,78\%), 12 Unidade de Cardiologia Intensiva (UCI) (1,63\%), 13 - UTI de Recém-nascidos (1,48\%), 14 - PA Pediátrico (1,33\%), 15 - Centro de Tratamento da Criança com Câncer (CTCriaC) (1,33\%).

Entre as amostras coletadas, foram identificados 40 diferentes microrganismos. A relação dos microrganismos encontrados está demonstrada na Tabela 1.

Tabela 1. Prevalência de microrganismos isolados de secreções de pele e tecidos moles no hospital terciário, no período de janeiro de 2014 a dezembro de 2017.

\begin{tabular}{lc}
\hline MICRORGANISMO & QUANTIDADE x/675 e \% \\
\hline Staphylococcus aureus & $118-17,48 \%$ \\
Pseudomonas aeruginosa & $106-15,70 \%$ \\
Staphylococcus epidermidis & $65-9,63 \%$ \\
Escherichia coli & $60-8,89 \%$ \\
Acinetobacter baumanii complex & $51-7,55 \%$ \\
Enterococcus faecalis & $47-6,96 \%$ \\
Klebsiella pneumoniae ssp pneumoniae & $37-5,48 \%$ \\
Proteus mirabilis & $31-4,59 \%$ \\
Staphylococcus haemolyticus & $20-2,96 \%$ \\
Enterobacter cloacae & $16-4,27 \%$ \\
Enterobacter cloacae ssp dissolvens & $13-1,92 \%$ \\
Morganella morganii ssp morganii & $17-2,52 \%$ \\
Serratia marcescens & $12-1,78 \%$ \\
Enterococcus faecium & $11-1,63 \%$ \\
Enterobacter aerogenes & $8-1,18 \%$ \\
Proteus vulgaris group/ Proteus penneri & $7-1,04 \%$ \\
Klebsiella oxytoca & $5-0,74 \%$ \\
Staphylococcus xylosus & $5-0,74 \%$ \\
Staphylococcus saprophyticus & $5-0,74 \%$ \\
Staphylococcus capitis & $5-0,74 \%$ \\
Staphylococcus lentus & $4-0,59 \%$ \\
Citrobacter freundii & $4-0,59 \%$ \\
Enterococcus spp. & $3-0,44 \%$ \\
Staphylococcus cohnii ssp urealyticus & $3-0,44 \%$ \\
Streptococcus agalactiae & $2-0,30 \%$ \\
Hafnia alvei & $2-0,30 \%$ \\
Enterococcus gallinarum & $2-0,30 \%$ \\
Staphylococcus hominis ssp hominis & $2-0,30 \%$ \\
Staphylococcus intermedius & $2-0,30 \%$ \\
Citrobacter brakii & $2-0,30 \%$ \\
Outros microrganismos & $10-1,48 \%$ \\
\hline Font:Auta 2020 & \\
\hline
\end{tabular}

Fonte: Autora, 2020. 
Observação - Tabela 1: Outros microrganismos compreende-se por: Serratia liquefaciens group, Stenotrophomonas maltophilia, Aeromonas hydrophila/caviae, Enterobacter cancerogenus, Citrobacter koseri, Sphingomonas paucimobilis, Citrobacter youngae, Staphylococcus lugdunensis, Serratia fonticola e Edwardsiella tarda.

A relação do perfil de resistência aos antimicrobianos dos microrganismos prevalentes está descrita na Tabela 2.

Tabela 2. Perfil de resistência microbiana dos microrganismos prevalentes provenientes de infecção de pele e tecidos moles, em hospital terciário, janeiro de 2014 a dezembro de 2017.

\begin{tabular}{|c|c|c|c|c|}
\hline \multirow[t]{2}{*}{ Antimicrobianos } & \multicolumn{4}{|c|}{ Resistência N (\%) } \\
\hline & $\begin{array}{l}\text { Staphylococcus } \\
\text { aureus (118) }\end{array}$ & $\begin{array}{l}\text { Pseudomonas } \\
\text { aeruginosa (106) }\end{array}$ & $\begin{array}{l}\text { Staphylococcus } \\
\text { epidermidis (65) }\end{array}$ & $\begin{array}{l}\text { Escherichia } \\
\text { coli }(60)\end{array}$ \\
\hline Eritromicina & $33(27,97)$ & - & $50(76,92)$ & - \\
\hline Clindamicina & $43(36,44)$ & - & $39(60,00)$ & - \\
\hline Oxacilina & $29(24,58)$ & - & $50(76,92)$ & \\
\hline Ciprofloxacino & - & $48(45,28)$ & - & $12(20,00)$ \\
\hline Imipenem & - & $57(53,77)$ & - & 0 \\
\hline Cefepima & - & $36(33,96)$ & - & $14(23,33)$ \\
\hline Ceftazidima & - & $34(32,07)$ & - & $13(21,67)$ \\
\hline Meropenem & - & $47(44,34)$ & - & 0 \\
\hline Gentamicina & - & $42(39,62)$ & - & $5(8,33)$ \\
\hline Trimetoprima + Sulfametoxazol & $1(0,85)$ & - & $40(61,54)$ & - \\
\hline Piperacilina/Tazobactam & - & $32(30,19)$ & - & $6(10,00)$ \\
\hline Ampicilina & - & - & - & $38(63,33)$ \\
\hline Ampicilina / Sulbactam & - & - & - & $28(46,67)$ \\
\hline Amicacina & - & $16(15,09)$ & - & 0 \\
\hline Ceftriaxona & - & - & - & $14(23,33)$ \\
\hline Cefuroxina & - & - & - & $13(21,67)$ \\
\hline Rifampicina & 0 & - & $2(3,08)$ & - \\
\hline Ertapenem & - & - & - & 0 \\
\hline Vancomicina & 0 & - & 0 & - \\
\hline Linezolida & 0 & - & 0 & - \\
\hline
\end{tabular}

Fonte: Autora, 2020.

\section{DISCUSSÃO}

No período analisado, 56,15\% das amostras foram correspondentes a pacientes do gênero masculino concordando com estudo realizado por Silva e colaboradores, na cidade de Passo Fundo (Rio Grande do Sul, Brasil), o qual também encontrou predomínio do gênero masculino, com uma porcentagem de $75 \%{ }^{7}$. Dos materiais clínicos encaminhados, 0 
maior isolamento foi o de feridas cirúrgicas $(61,48 \%)$. Tornam-se necessárias medidas preventivas, educacionais e de controle para reduzir e controlar sua incidência, por meio de um processo de conscientização coletiva, as quais vão reverter em taxas de infecção em limites desejáveis para cada tipo de procedimento cirúrgico ${ }^{8}$. As infecções do sítio cirúrgico acarretam grandes encargos sócio-econômicos às instituições em decorrência dos altos custos hospitalares, além de representar um agravo com múltiplos fatores envolvidos. Assim, evidenciando a importância do resultado obtido neste trabalho, o qual teve um grande número de isolamentos de feridas cirúrgicas.

Referente às unidades hospitalares, a Clínica Cirúrgica foi o setor hospitalar onde houve o maior número isolamentos, com $20,89 \%$ de amostras, dado este que confirma a prevalência de feridas cirúrgicas como sendo principal material clínico encontrado. 0 desenvolvimento da infecção no local cirúrgico resulta em morbidade significativa do paciente, sendo que a infecção pós-operatória continua a ser a complicação mais comum dos procedimentos cirúrgicos ${ }^{9}$.

Dentre os microrganismos isolados de secreções de pele e tecidos moles, o prevalente foi Staphylococcus aureus, totalizando 118 amostras (17,48\%). Esse resultado está de acordo com estudo realizado em 2015 por Souza e colaboradores em Minas Gerais, o qual encontrou predomínio de 21,2\% para esta bactéria ${ }^{10}$. Este dado é significativo uma vez que esta bactéria é responsável por infecção necrosante da pele e tecidos moles ${ }^{11}$.

Referente ao perfil de resistência, foram avaliados os antimicrobianos classes Ae B para os quatro microrganismos prevalentes ${ }^{12}$. Relacionado ao S. aureus, $36,44 \%$ apresentaram resistência à clindamicina, tal medicamento é usado frente anaeróbicos e com frequência em pomadas para uso tópico ${ }^{13}$. A clindamicina é um importante agente terapêutico para as infecções por MRSA, principalmente em infecções de tecidos moles. No entanto, a resistência a macrolídeos, lincosamida e estreptogramina B (MLSB) induzível (iMLSB) tem conferido falhas terapêuticas ao uso desse antimicrobiano, com consequente aumento da mortalidade e do tempo de internação dos pacientes ${ }^{14,15}$.

Os isolados de S. aureus e S. epidermidis não apresentaram resistência frente à vancomicina e linezolida. São raras as resistências reportadas frente a esses agentes antimicrobianos ${ }^{16}$. Quando intermediários e ou resistência for detectada, recomenda-se que sejam enviados a centros de referência para confirmação ${ }^{12}$.

Em relação à Pseudomonas aeruginosa, 53,77\% das cepas apresentaram resistência ao imipenem e 44,34\% ao meropenem. Atualmente, as opções terapêuticas para o tratamento das infecções causadas por esse microrganismo são limitadas, muitas vezes restringindo-se ao uso de carbapenêmicos. Portanto, a resistência a estes antimicrobianos é uma questão de saúde pública, uma vez que são empregados como último recurso no tratamento de infecções graves de origem hospitalar, causadas por bactérias Gram-negativas multirresistentes. No Brasil, os principais mecanismos relacionados com fenótipos multirresistentes de P. aeruginosa são produção de metalo-beta-lactamases (MBLs), perda de porina e superexpressão de bombas de efluxo, o que pode explicar os altos índices de resistência a carbapenêmicos. A emergência de cepas com essas características é preocupante, tendo em vista a escassez de terapias efetivas no 
tratamento de infecções por esse patógeno ${ }^{17}$.

Entre os Staphylococcus epidermidis $76,92 \%$ apresentaram resistência à oxacilina. Estes microrganismos normalmente são colonizantes da pele, por isso advém seu nome, sendo patogênico somente para indivíduos imunocomprometidos e institucionalizados. Preocupante é o fato da grande percentagem encontrada neste estudo de isolados resistentes à oxacilina, uma vez que eles apresentam resistência a todos os antibióticos beta-lactâmicos ${ }^{16,18}$.

Em relação a E. coli, 63,33\% apresentaram resistência à ampicilina. Kadosaki e colaboradores, em 2012, realizaram a análise do uso e da resistência bacteriana aos antimicrobianos em nível hospitalar. Em seu estudo, foi relatada a resistência de $67,20 \%$ a este antimicrobiano ${ }^{19}$.

\section{CONSIDERAÇÕES FINAIS}

Estes resultados demostram que a prevalência de microrganismos envolvidos nas infecções de pele e tecidos moles neste nosocômio é o mesmo do citado na literatura internacional ${ }^{6}$. Fato preocupante encontrado em nosso estudo é o da $P$. aeruginosa ser o $2^{\circ}$ agente mais isolado. Além de apresentar significativa resistência frente aos carbapenêmicos, uma vez que, como é conhecido, ele está envolvido na maioria de infecções crônicas. Esse fato evidencia a necessidade de vigilância epidemiológica com o objetivo de controle da resistência bacteriana. Portanto, estar ciente do perfil epidemiológico e de resistência é extremamente importante para ser instituída uma antibioticoterapia adequada aos pacientes acometidos por infecções de pele e tecidos moles.

\section{REFERÊNCIAS}

1. Simões CMSB. Infecções Hospitalares Bacterianas no Século XXI. Porto (Portugal): Faculdade de Ciências da Saúde; 2016.

2. Giarola LB, Baratieri T, Costa AM, et al. Infecção Hospitalar na Perspectiva dos Profissionais de Enfermagem: Um Estudo Bibliográfico. Cogitare Enferm. 2012; 17:151-157.

3. Kempfer CB, Hörner R, Tizotti MK, et al. Culturas de Secreções de Pele: Estudo de Prevalência e Sensibilidade aos Antimicrobianos em um Hospital Universitário. Saúde (S. Maria). 2010; 36:57-66.

4. Agência Nacional de Vigilância Sanitária (BR). Medidas de Prevenção de Infecção Relacionada à Assis- 
tência à Saúde. Capítulo 1: Medidas de Prevenção de Pneumonia Associada à Assistência à Saúde. Brasília: Agência Nacional de Vigilância Sanitária; 2017.

5. Cardona AF, Wilson SE. Skin and soft-tissue infections: a critical review and the role of telavancin in their treatment. Clin Infect Dis. 2015; 61:69-78.

6. Society for Healthcare Epidemiology of America/ Infectious Diseases Society of America. Practice Recommendation - Strategies to prevent surgical site infections in acute care hospitals: 2014 update. Infect Control Hosp Epidemiol. 2014; 35:605-627.

7. Silva MGC, Rodrigues GS, Gonçalves IL, et al. Candida species distribution and fluconazole susceptibility of blood isolates at a regional hospital in Passo Fundo, RS, Brasil. J Bras Patol Med Lab. 2015; 51:158-161 ᄀ.

8. Khan HA, Ahmad A, Mehboob R. Nosocomial infections and their control strategies. Asian Pac J Trop Biomed. 2015; 5:509-514.

9. Lachiewicz MP, Moulton LJ, Jaiyeoba O. Pelvic Surgical Site Infections in Gynecologic Surgery. Infect Dis Obstet Gynecol. 2015.

10. Souza GAAD, Garcia LM, Rocha SS, et al. Perfil Microbiológico de infecções de pele e partes moles em pacientes internos de um hospital universitário. R Epidemiol Control Infec. 2016; 6:33-36.

11. Liu Y, Xu Z, Yang Z, et al. Characterization of community-associated Staphylococcus aureus from skin and soft-tissue infections: a multicenter study in China. Emerg Microbes Infect. 2016.

12. Clinical Laboratory Standards Institute. Performance standards for antimicrobial disk suscrptibility tests; Approved standards. CLSI; 2016.

13. Santos JB, Almeida OLS, Silva LM, et al. Eficácia da combinação tópica de peróxido de benzoíla 5\% e clindamicina $1 \%$ para o tratamento da hipomelanose macular progressiva: um estudo randomizado, duplo-cego, placebo-controlado. Na Bras Dermatol. 2011; 86:50-54. 
14. Silva ACO, Silva RCG, Oliveira SR. Resistência microbiana à clindamicina em isolados clínicos de Staphylococcus sp. provenientes de hemoculturas de pacientes hospitalizados. J Bras Patol Med Lab. 2016; 52(3):165-170.

15. Castellano MG, Perozo MA, Molero CMJ, et al. Resistência a lá clindamicina inducida por eritromicina en cepas de Staphylococcus aureus de origen clínico. Kasmera. 2015; 43(1):13-15.

16. Brazilian Committee on Antimicrobial Susceptibility Testing. Tabelas de pontos de corte para interpretação de CIMs e diâmetros de halos. BrCAST; 2017.

17. Dantas RCC, Silva RTE, Ferreira ML, et al. Molecular epidemiological survey of bacteremia by multidrug resistant Pseudomonas aeruginosa: the relevance of intrinsic resistance mechanisms. PLoS ONE. 2017.

18. Vilefort LOR, Vasconcelos LSNOL, Costa DM, et al. Colonização de trabalhadores de áreas de apoio hospitalar por Staphylococcus sp.: aspectos epidemiológicos e microbiológicos. Rev Eletr Enf. 2015.

19. Kadosaki LL, Sousa SF, Borges CM. Análise do uso e da resistência bacteriana aos antimicrobianos em nível hospitalar. Ver Bras Farm. 2012; 93:128-135. 